\title{
Optimized Wavelength-Sized Scalar Horns as Antenna Radiation Standards
}

\author{
ARTHUR J. ESTIN, SENIOR MEMBER, IEEE, CARL F. STUBENRAUCH, MEMBER, IEEE, \\ ANDREW G. REPJAR, MEMBER, IEEE, AND ALLEN C. NEWELL, MEMBER, IEEE
}

\begin{abstract}
The properties of beamwidth, directivity, and polarization of wavelength-size scalar horns are analyzed and optimized theoretically and confirmed experimentally to determine the usefulness of such horns as standards. Agreement between theoretical predictions and measurements was good.
\end{abstract}

\section{INTRODUCTION}

$\mathrm{T}$ HE "scalar" electromagnetic horn, usually of circular cross section, was so named [1] because it grooved wall presents the same boundary conditions to all polarizations and it has a tapered field distribution that is axially symmetric. It is also sometimes ca'led the "corrugated" horn because of the circumferentially grooved interior.

Various workers had realized that the radiation characteristics of a horn can be modified in desirable ways, such as reduced sidelobe levels, equalized $E$ - and $H$-plane beamwidths, and greater axial symmetry, by simultaneously using more than one waveguide mode for excitation of the horn. Love [2] used a square cross section excited in the $\mathrm{TE}_{10}$ and $T E_{01}$ modes. Potter [3] used the circular $\mathrm{TE}_{11}$ and $\mathrm{TM}_{11}$ modes, which achieve to a very high degree all of the properties mentioned above. The difficulty with Potter's design was that achieving precise amplitude and phase balance of the two differentially dispersive modes was difficult, at best, and impossible over a significant bandwidth. It was realized simultaneously in Australia [4], [5] and the United States [1], [6] that these modes could be "locked together" in the $\mathrm{HE}_{11}$ hybrid mode configuration by imposing an anisotropic boundary condition on the excitation structure leading to the aperture. A bandwidth of about 1.7:1 was now readily attainable primarily because of the fact that the $\mathrm{HE}_{11}$ is the fundamental hybrid mode propagated in the anisotropic waveguide and the next higher mode $\left(\mathrm{EH}_{11}\right)$ has a much higher cutoff frequency [7].

By virtue of these various properties-low sidelobes, quasi-Gaussian skirt shape, axial symmetry, wide useful frequency band, etc.--the scalar horn has become well recognized as an efficient feed device for large paraboloidal reflector

Manuscript received October 6, 1981. This work was supported by the U.S. Air Force Satellite Control Facility, Sunnyvale, CA.

A. J. Estin was with the National Bureau of Standards, Boulder, CO. He is now a Consulting Engineer, Paonia, $\mathrm{CO}$.

C. F. Stubenrauch, A. G. Repjar, and A. C. Newell are with the Electromagnetic Fields Division, National Bureau of Standards, Boulder, CO 80302 .

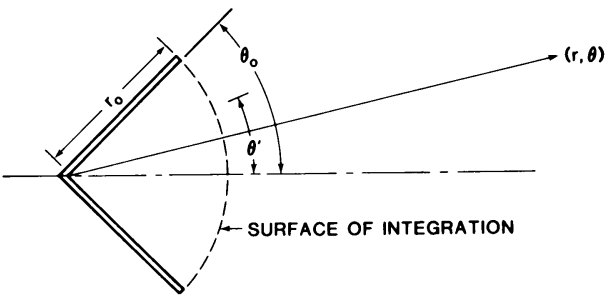

Fig. 1. Diagram of circular horn antenna showing slant length $r_{0}$, flare angle $\theta_{0}$, and surface of integration for field calculations.

antennas and as a desirable antenna range and antenna comparison standard. The purpose of this paper is to point out that such horns need not be electrically large to be useful and that the size parameters, slant length, and flare angle can be optimized for minimization of any desired beamwidth contour or for maximization of directivity. Although one cannot, in general, achieve these conditions simultaneously, it is found that they can be nearly achieved. This is somewhat analogous to attempting to achieve simultaneous maximum power transfer and minimum reflection in a network having a reactive input impedance component.

\section{BACKGROUND THEORY}

The radiation characteristics of the scalar horn have been analyzed in terms of spherical modes, which are applicable to arbitrarily wide flare angles [8], [9]. Under the assumption that an unperturbed spherical $\mathrm{HE}_{11}$ waveguide mode illuminates the radiating aperture of the scalar horn, the following expressions can be obtained for the far fields by a KirchhoffHuygens integration over a spherical cap enclosing the horn [3]:

$$
E_{\theta}=-A\left(r_{0}\right) \frac{e^{-j k r}}{r} F\left(\theta, \theta_{0}, r_{0}\right) \cos \phi
$$

and

$$
E_{\phi}=A\left(r_{0}\right) \frac{e^{-j k r}}{r} F\left(\theta, \theta_{0}, r_{0}\right) \sin \phi .
$$

Referring to Fig. $1, r_{0}$ and $\theta_{0}$ are the slant length and flare angle, respectively, of the horn; $r, \theta$, and $\phi$ are the spherical coordinates of the field point; and $\theta^{\prime}$ is the coordinate of integration.

The pattern function $F\left(\theta, \theta_{0}, r_{0}\right)$ can be reduced to a single integration which must be evaluated numerically. 


$$
\begin{aligned}
F\left(\theta, \theta_{0}, r_{0}\right)= & \int_{0}^{\theta_{0}}\left\{\left(\cos \theta+\cos \theta^{\prime}\right)\left[J_{0}(y)+J_{2}(y)\right]\right. \\
& +\left(1+\cos \theta \cos \theta^{\prime}\right)\left[J_{0}(y)-J_{2}(y)\right] \\
& \left.+2 j \sin \theta \sin \theta^{\prime} J_{1}(y)\right\} f_{\nu}^{1}\left(\theta^{\prime}\right) \\
& \cdot \exp (j x) \sin \theta^{\prime} d \theta^{\prime}
\end{aligned}
$$

where

$$
\begin{aligned}
& x=k r_{0} \cos \theta \cos \theta^{\prime} \\
& y=k r_{0} \sin \theta \sin \theta^{\prime}
\end{aligned}
$$

$J_{i}(y)$ is the Bessel function of first kind and order $i$

$$
f_{\nu}^{1}\left(\theta^{\prime}\right)=\frac{d P_{\nu}^{1}\left(\cos \theta^{\prime}\right)}{d \theta^{\prime}}+\frac{P_{\nu}^{1}\left(\cos \theta^{\prime}\right)}{\sin \theta^{\prime}}
$$

and $P_{\nu}^{1}\left(\cos \theta^{\prime}\right)$ is the associated Legendre function of order 1 and degree $\nu$.

The value of $\nu$ is an implicit function of $\theta_{0}$ and must be determined from a characteristic equation as discussed in the references.

The pattern function was calculated on a large computer for field angles $0(5) 90^{\circ}$, for flare angles $25(5) 60^{\circ}$, and for slant length/wavelength ratio $0.8(0.05) 6$. The integration was by means of Simpson's Rule with an interval of $1^{\circ}$. The results of this calculation were transferred to a programmable desk calculator for subsequent use.

Directivity calculations were obtained by numerical evaluation of

$$
G=10 \log \left\{\frac{2\left|F\left(0, \theta_{0}, r_{0}\right)\right|^{2}}{\int_{0}^{\pi}\left|F\left(\theta, \theta_{0}, r_{0}\right)\right|^{2} \sin \theta d \theta}\right\} .
$$

The principal contribution to the far side and back radiation is edge diffraction from the horn, which is neglected in this calculation of the fields. Accordingly, a worst case limit was assumed in the evaluation of (4) by using $F\left(\pi / 2, \theta_{0}, r_{0}\right)$ for all values of $F\left(\theta, \theta_{0}, r_{0}\right)$ where $\pi / 2<\theta \leq \pi$.

\section{Theoretical Results}

The semibeamwidths of selected contours were determined by applying a search-and-interpolate routine to the patterns which were obtained as in the previous section. These were plotted as functions of slant length (normalized to wavelength) over a range of 0.8 to 2.0 , using the flare angle as a parameter. Fig. 2 shows these results for the $-10-\mathrm{dB}$ contour. Since minimum beamwidth is usually a desired property, it is important to note that the three-dimensional surface representing beamwidth as a function of flare angle and slant length passes through a minimum for each slant length. This minimum represents the optimum flare angle for that slant length, which varies from somewhat over $60^{\circ}$ for a slant length of $0.8 \lambda$ to about $40^{\circ}$ at $2 \lambda$. The optimum flare angle is somewhat smaller for the $-15-,-20-$, or $-25-\mathrm{dB}$ contours. A three-point Lagrangian interpolation was used to find the loci of these minima, and the results are shown in Fig. 3.

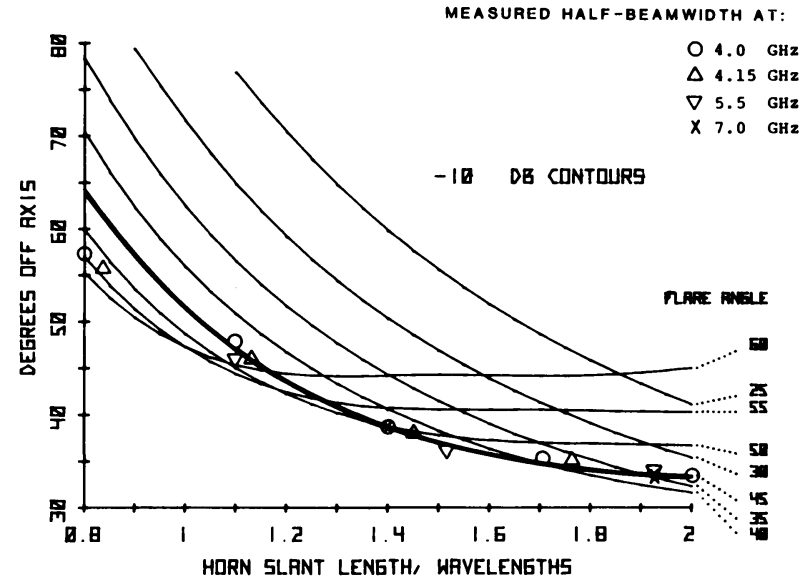

Fig. 2. Location of $-10-\mathrm{dB}$ beam contour as a function of slant length for various flare angles showing confirmation of theoretical predictions by experimental measurements.

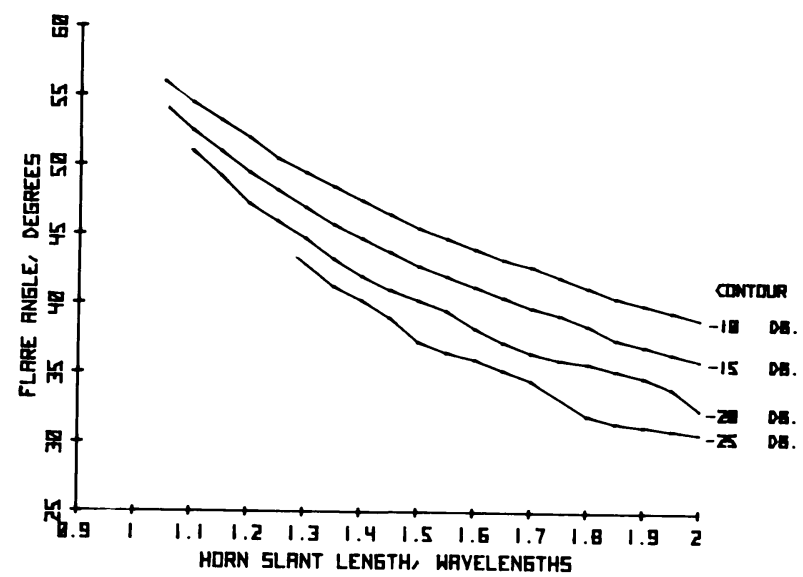

Fig. 3. Optimum horn dimensions giving minimum beamwidths at $-10-$, $-15-,-20-$, and $-25-\mathrm{dB}$ levels.

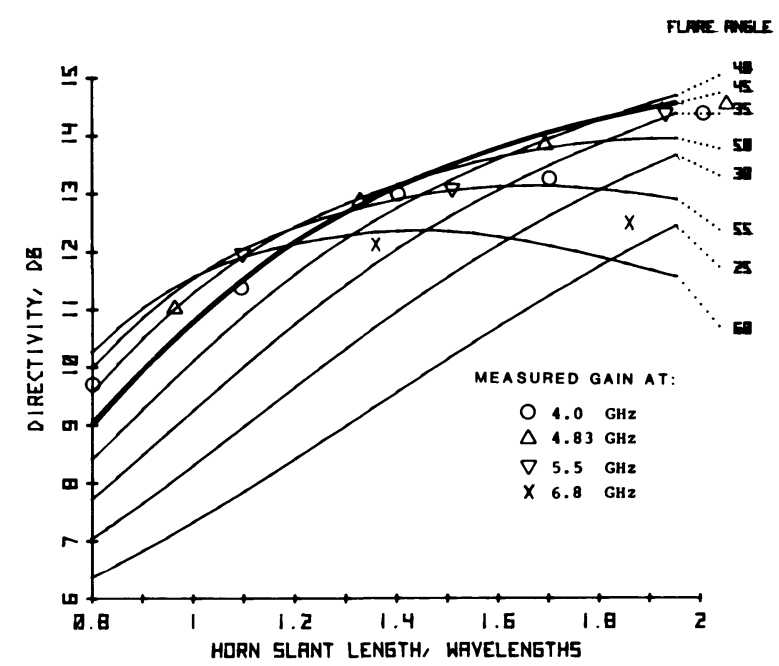

Fig. 4. Horn directivity as a function of slant length for various flare angles, as well as experimentally determined gain values.

A second major radiation characteristic is the directivity. Fig. 4 shows the directivity plotted as a function of slant length, with flare angle as a parameter. This directivity passes through 


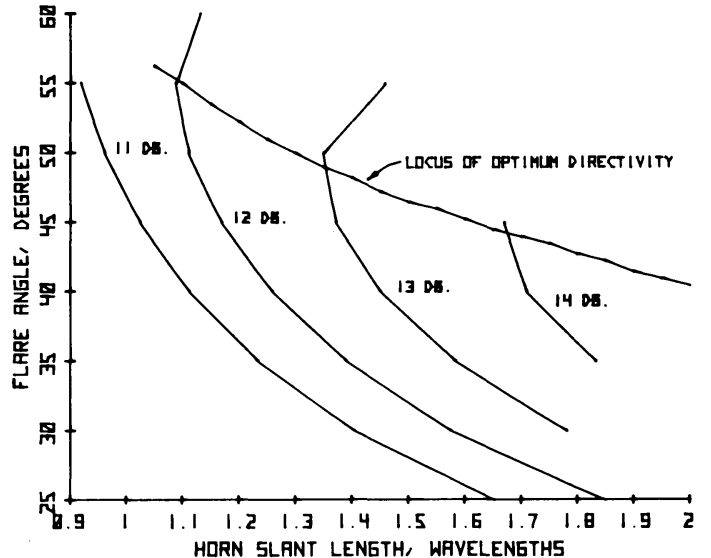

Fig. 5. Contours of constant directivity for scalar horns as a function of flare angle and slant length. Locus of optimum directivity gives maximum directivity obtainable for a given slant length.

a maximum for each slant length. Fig. 5 shows the locus of the antenna dimensions for maximum directivity and, in addition, gives constant directivity contours so that the degradation in directivity can be estimated for nonoptimum dimensions.

\section{EXPERIMENTAL RESULTS}

A scalar horn having a $45^{\circ}$ flare angle and five interchangeable apertures was designed and constructed. At the design frequency of $4 \mathrm{GHz}$, these apertures correspond to slant lengths of $0.8,1.1,1.4,1.7$, and 2.0 wavelengths, and are designated as \#0,\#1,, , \# 4, respectively. The design frequency is that frequency for which the groove depth is slightly over a quarter wavelength and the surface reactance approaches negative infinity. The desired $\mathrm{HE}_{11}$ hybrid mode can be maintained as long as the surface reactance is negative, so the theoretical maximum bandwidth extends from the design frequency to nearly twice that frequency. In practice, a bandwidth of about 1.7 or 1.8 can be achieved before the balanced hybrid mode approximation becomes so poor that the desired radiation characteristics are degraded. Groove periodicity was set at $0.15 \lambda$ at $4 \mathrm{GHz}$ [10], and the ratio of gap width to corrugation period was 0.8 which is somewhat larger than usual for ease in machining. Excitation will not be discussed in this paper.

Measurements were made on the test horn assembly over the frequency range of 4 to about $7 \mathrm{GHz}$, a bandwidth of 1.75 . Three types of measurements were made: input impedance, which will not be discussed here because it relates primarily to the design of mode excitation and coupling structures; axial gain and polarization using the NBS Near-Field Extrapolation Range; and field patterns, which were made on a model mount in an indoor far-field range. The patterns were measured by taking $E$-plane, $H$-plane, and $45^{\circ}$ cuts to beyond $90^{\circ}$ off axis for the \#0, \#2, and \#4 apertures at 4, 4.15, 5.5, and $7 \mathrm{GHz}$. In addition, $E$-plane cuts were made at the same frequencies for the \#1 and \#3 apertures.

In order to obtain a measure of departures from axial symmetry, each set of three half-beamwidths (obtained from the $E$-plane, $H$-plane, and $45^{\circ}$ cuts) was treated as a statistical ensemble. Its standard deviation was a measure of axial asymmetry, and the average of the three angles was compared with the theoretical beamwidth. The asymmetry was found in this way to be about $1^{\circ}$ at the design and midband frequencies of 4 and $5.5 \mathrm{GHz}$, respectively. At $7 \mathrm{GHz}$, the asymmetry was $2^{\circ}$ to $3^{\circ}$ for the \#2 and \#4 apertures and about $5^{\circ}$ to $7^{\circ}$ for the \#0 aperture.

The averages of the half-beamwidths of the three cuts for $\# 0$, \#2, and \#4, and the value for the $E$-plane cut for \#1. and \#3 were compared to theoretical calculations. Depending upon frequency and aperture, the 10-dB half-beamwidths vary between about $35^{\circ}$ and $60^{\circ}$ and the 20 -dB half-beamwidths from $50^{\circ}$ to greater than $90^{\circ}$. The differences between calculated and measured beamwidths for \# 1, \#2, \#3, and \#4 were less than $1^{\circ}$ at design frequency, increase to $1^{\circ}$ to $2^{\circ}$ at midband, and increase further to $4^{\circ}$ to $6^{\circ}$ at $7 \mathrm{GHz}$. The -10- $\mathrm{dB}$ contour differences for the \# 0 aperture had the same general shape of frequency dependence, but were about $7^{\circ}$ narrower then calculated. A reason for this will be discussed later. The experimental values for these -10 -dB half-beamwidths are plotted in Fig. 2, and are keyed for frequency. For ease in comparison with theory, the curve corresponding to a $45^{\circ}$ flare angle has been emphasized.

Cross-polarization profiles were taken by rotating the antenna axially by $90^{\circ}$ and $270^{\circ}$ with respect to the test probe. This does not, in general, give a true cross polarization, but does constitute an upper bound. In the principal plane cuts, this upper bound was never greater than $-30 \mathrm{~dB}$, and usually less than $-35 \mathrm{~dB}$ with respect to boresight copolarization. In the $45^{\circ}$ cuts, which normally have the worst cross polarization; this upper bound increased to about $-30 \mathrm{~dB}$ for design and midband frequencies and to between -17 to $-22 \mathrm{~dB}$ at $7 \mathrm{GHz}$. The off-axis angle for which the worst cross polarization occurred was usually between $40^{\circ}$ and $60^{\circ}$ and in no case was less than $22^{\circ}$. Accurate measurements of the on-axis cross polarization made with the near-field extrapolation technique [11] indicate that the cross polarization is at least 55 to $60 \mathrm{~dB}$ down for the design and midband frequencies, and at least 45 $\mathrm{dB}$ down at the high end of the band.

Experimental values for the gain were also obtained by extrapolation measurements at $4.0,4.83,5.5$, and $6.8 \mathrm{GHz}$. These values are plotted in Fig. 4 and, again, the $45^{\circ}$ flare angle curve is emphasized. Values for the low and midband frequencies fall within $0.5 \mathrm{~dB}$ of calculations, which is nearly as good as can be achieved with pyramidal horns. The values of $6.8 \mathrm{GHz}$, however, fall considerably below the calculated curve-an effect probably attributable to departure from mode balance at the band limit. The phase center of the antenna was always well defined and was about $2 \mathrm{~cm}$ in front of the apex of the cone.

\section{CONCLUSION}

We have shown (Fig. 2) that a scalar horn having an aperture of the order of one wavelength does, in fact, have the radiation properties predicted for it by a spherical far-field analysis that does not include edge diffraction effects. There are two limitations to this conclusion. Departures from theo- 
retical predictions become significant at about 1.75 times design frequency, and also for a very short slant length of $0.8 \lambda$. This latter effect may have been caused in part by a somewhat different design limitation. The horn does not really form a complete cone, but, in fact, is a cone which is truncated by the cylindrical waveguide which excites it. Thus the actual antenna slant length as measured from the aperture edge to the intersection with the feed waveguide for the \#0 aperture was only about 25 percent of the distance from the edge to the apex of the hypothetical cone. It is, therefore, possible that the spherical waveguide mode may not have been well established before the wave was launched at the aperture. Even with this limitation, the agreement between various measurements of the \#0 aperture with calculations is quite reasonable.

We thus conclude that the performance of electrically small scalar horns can be predicted rather well, and they do possess the features desired for reliable antenna test standards.

\section{ACKNOWLEDGMENT}

The help and encouragement of Dr. R. C. Baird is appreciated. Helpful discussions with Dr. D. C. Hogg are also acknowledged. D. P. Kremer performed the measurements.

\section{REFERENCES}

[1] A. F. Kay, "The scalar feed," AFCRL Rep. 64-347, Mar. 1964.

[2] A. W. Love, "The diagonal antenna," Microwave J., vol. V, pp. 117-122, Mar. 1962.

[3] P. D. Potter, "A new horn antenna with suppressed sidelobes and equal bandwidths," Microwave J., vol. VI, pp. 71-78, June 1963.

[4] H. C. Minnett and B. MacA. Thomas, "A method of synthesizing radiation patterns with axial symmetry, IEEE Trans. Antennas Propagat., vol. AP-14, pp. 654-656, Sept. 1966.

[5] V. H. Rumsey, "Horn antennas with uniform power patterns around their axes," IEEE Trans. Antennas Propagat., vol. AP-14, pp. 656-658, Sept. 1966.

[6] R. E. Lawrie and L. Peters, Jr., "Modification of horn antennas for low sidelobe levels," IEEE Trans. Antennas Propagat., vol. AP-14, pp. 605-610, Sept. 1966.

[7] P. J. B. Clarricoats, “Analysis of spherical hybrid modes on a corrugated conical horn," Electron. Lett., vol. 5, pp. 189-190, May 1, 1969.

[8] P. J. B. Clarricoats and P. K. Saha, "Propagation and radiation behavior of corrugated feeds: Part 2-Corrugated-conical-horn feed," Proc. Inst. Elec. Eng., vol. 118, pp. 1177-1186, Sept. 1971.

[9] J. K. M. Jansen, M. E. J. Jeuken, and C. W. Lambrechtse, "The scalar feed,” Arch. Elek, Übertragung, vol. 26, pp. 22-30, Jan. 1972.

[10] C. A. Mentzer and L. Peters, Jr. "Properties of cutoff corrugated surfaces for corrugated horn design," IEEE Trans. Antennas Propagat., vol. AP-22, pp. 191-196, Mar. 1974.

[11] A. C. Newell, R. C. Baird, and P. F. Wacker, “Accurate measurement of antenna gain and polarization at reduced distances by an extrapolation technique," IEEE Trans. Antennas Propagat., vol. AP-21, pp. 418-431, July 1973.

\section{Skew Estimator Method for Alignment of Troposcatter Antennas and Synchronization of Master Clocks}

JAY A. WEITZEN, A. BURR FONTAINE, MEMBER, IEEE, AND WILLIAM P. BIRKEMEIER, MEMBER, IEEE

\begin{abstract}
Microprocessor-based instrumentation for optimum alignment of troposcatter antennas and simultaneous synchronization of transmitter and receiver oscillators is discussed. The instrument estimates various spectral moments of the Doppler spectrum of the received signal. When the third central moment or skew is zero, the antennas are properly aligned. Tests of this method indicate that alignment to within $\frac{1}{10}$ beamwidth within a time period of $10 \mathrm{~min}$ or less is possible.
\end{abstract}

Manuscript received September 24, 1981. This work was supported by the US Air Force under Contract F 30602-77-C-0148.

The authors are with the Department of Electrical and Computer Engineering, University of Wisconsin, Madison, WI 53706.

\section{INTRODUCTION}

$\mathrm{U}$ NTIL RECENTLY, azimuthal alignment of troposcatter antennas has been attempted by swinging the beams so as to maximize the received power. Unfortunately, finding the proper pointing angle is time consuming and often inaccurate because of deep and persistent fading in the channel. In earlier work, Birkemeier and Sill [1] established a new technique for alignment which uses the fact that cross-path winds create a Doppler-spread spectrum. A monochromatic signal is transmitted and the shape of the received Doppler spectrum is used to determine alignment. Their method in- 